\title{
ON-LOOM, REAL-TIME, NONCONTACT DETECTION OF FABRIC DEFECTS BY ULTRASONIC IMAGING*
}

\author{
H.-T. Chien, S.-H. Sheen, W. P. Lawrence, \\ K. Razazian ${ }^{\dagger}$, and A. C. Raptis
}

\section{Energy Technology Division ARGONNE NATIONAL LABORATORY Argonne, Illinois 60439}

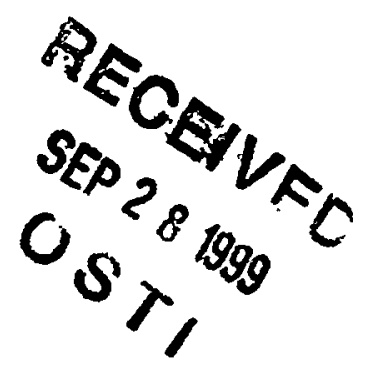

The submitted manuscript has been authored by a contractor of the U.S. Government under contract No. W-31-109-ENG-38. Accordingly, the U.S. Government retains a nonexclusive, royalty-free license to publish or reproduce the published form of this contribution, or allow others to do so, for U.S. Government purposes.

August 1998

Final paper to be included in Proceedings of 25th Annual Review of Progress in Quantitative Nondestructive Evaluation, organized by the Center for NDE of the Institute for Physical Research and Technology at lowa State University, held at Snowbird, Utah, July 19-24, 1998.

'Work supported by U.S. Department of Energy, Office of Energy Research, Laboratory Technology Transfer Projects, under Contract W-31-109-ENG-38.

${ }^{\dagger}$ The current address is the Northern Telecom (Nortel) Corporation, 4100 Los Angeles Avenue, Simi Valley, CA 93063. 


\section{DISCLAIMER}

This report was prepared as an account of work sponsored by an agency of the United States Government. Neither the United States Government nor any agency thereof, nor any of their employees, make any warranty, express or implied, or assumes any legal liability or responsibility for the accuracy, completeness, or usefulness of any information, apparatus, product, or process disclosed, or represents that its use would not infringe privately owned rights. Reference herein to any specific commercial product, process, or service by trade name, trademark, manufacturer, or otherwise does not necessarily constitute or imply its endorsement, recommendation, or favoring by the United States Government or any agency thereof. The views and opinions of authors expressed herein do not necessarily state or reflect those of the United States Government or any agency thereof. 


\section{DISCLAIMER}

Portions of this document may be illegible in electronic image products. Images are produced from the best available original document. 
On-Loom, Real-Time, Noncontact Detection of Fabric Defects by Ultrasonic Imaging

H.-T. Chien, S.-H. Sheen, W. P. Lawrence, K. Razazian ${ }^{\dagger}$, and A. C. Raptis Energy Technology Division

Argonne National Laboratory

Argonne, IL 60439

\section{INTRODUCTION}

The textile industries of developed nations, such as the United States and the countries of western Europe, are now facing enormous competition from imported fabrics that are highly adaptable, quickly produced, less expensive, and of high quality. It is very important that the textile manufactures, as well as the machine manufactures, to manufacture a machine with high output, less waste, and individual-machine automation. One of the most labor-intensive tasks in textile production is the inspection and identification of fabric defects. Although visual inspection can identify most defects, an automated or semiautomated inspection system will be more reliable, eliminate potential human errors, improve quality control, reduce waste and energy consumption, and lower operating costs. Currently, an optic/visual system is commercially available, but it is an off-loom system and is very expensive. Other techniques, for example, ultrasonic, millimeter wave, capacitance, and photo-diode, have been studied. At Argonne National Laboratory (ANL), we developed a nonvisual, noncontact fabric-inspection system that can detects defects in real-time on the loom[1]. The system is compact, rugged, and low cost, requires minimal maintenance, is not sensitive to fabric color and vibration, and can easily be adapted to current loom configurations. Unlike other available techniques, the ANL ultrasonic system is not affected by vibration generated by the weaving machine.

The system is based on an ultrasonic imaging technique in which is used an array of ultrasonic transducer pairs that operate efficiently in air at a frequency of $0.5 \mathrm{MHz}$. Each pair consists of a focused transmitter and a flat-surface receiver that operate in a pitch-catch mode. The array rasters across the fabric in the pick direction at $10 \mathrm{in} . / \mathrm{sec}$. To greatly reduce the amount of collectable data, an electronic system analogically processes the signal of each channel before data acquisition. The latest digital signal processing (DSP) technique is used for real-time system control, data acquisition, and image processing. DSP-based algorithms are also developed to identify the locations, sizes, and types of

\footnotetext{
'The current address is the Northern Telecom (Nortel) Corporation, 4100 Los Angeles Avenue, Simi Valley, CA 93063.
} 
defects. This system has demonstrated its capability to detect the commonly generated fabric defects in both pick and warp directions, including missing picks, waste, oil spots, reed marks, end outs, moires, and misdraws.

\section{SYSTEM CONFIGURATION}

At ANL, a noncontact, on-loom ultrasonic inspection system was developed for real-time $100 \%$ defect inspection of fabrics. The system records all of the defect information and alerts operators whenever major defects occur; it also automatically determines defect size and location, and identifies and classifies defects. The system is an integration of five subsystems: an air-coupled transducer array, electronics, scanning mechanism, data acquisition, and imaging processing and defect analysis.

Figure 1 shows a prototype built for an air-jet loom, which has a nominal weaving speeds of 450 to 950 picks/min and can produce fabric with widths of $60-80 \mathrm{in}$. The nominal pick and warp density is $20-90$ picks/in. and 30-120 warps/in., i.e. the nominal size of the pick and warp yams is 0.011-0.050 in. and 0.008-0.033 in., respectively. The inspection system is based on the ANL ultrasonic imaging technique in which an array of ultrasonic transducer pairs operate efficiently in air at a frequency of $0.5 \mathrm{MHz}$. While scanning back and forth along the pick direction and feeding along the warp direction by the weaving machine, the system generates and analyzes a two-dimensional (2D) ultrasonic image every $30 \mathrm{sec}$ to detect, locate, measure, classify, and report defects. During scanning along the pick direction, more information is acquired for each pick but less for each warp. The resolution for detecting warp defects depends on scanning speed, repetition rate of the electronics, beam size of the transducer, separation distance of the transducer pair, and warp material and size. Nevertheless, it is very important to detect warp defects because most are running defects and must be corrected promptly before more waste is produced.

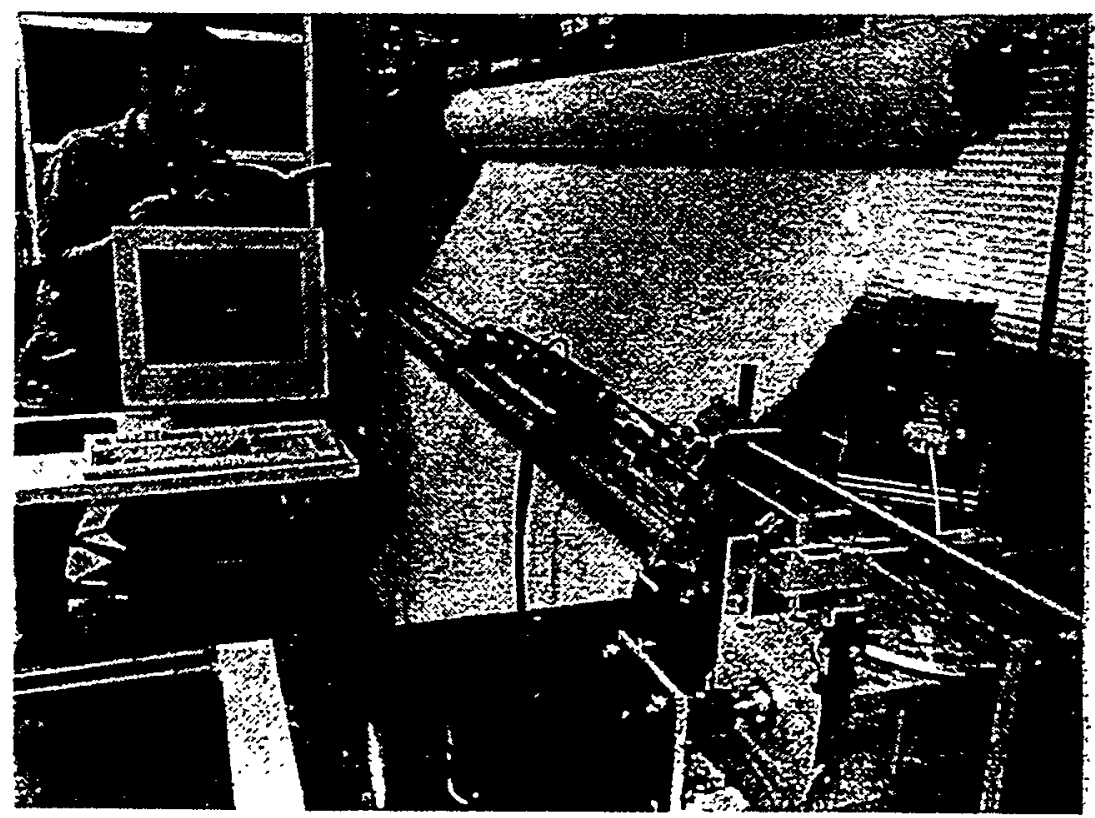

Figure 1. Prototype system for detecting fabric defects ultrasonically. 


\section{Air-Coupled Ultrasonic Transducer Array}

The most important task of this work is to design high power, extremely sensitive, low-cost air-coupled transducers for noncontact inspection of textiles. Both the pitch-catch and pulse-echo techniques were evaluated. The scanning setup of pulse-echo is simpler and less costly. However, to shorten the beam path, reduce transmitting energy loss, and avoid reflection signal complexity caused by machine vibration and multiechoes problems, the pitch-catch technique was selected, in which a transducer array of eight air-coupled pairs is operated at $0.5 \mathrm{MHz}$ for optimal balance between detection resolution and transducer energy. Both the transmitter and receiver are made of PZT-5H. The transmitter $1 \mathrm{in}$. in diameter and in focused length, respectively, with maximum power of $80 \mathrm{~V}$ and minimum beam size of $0.2 \mathrm{in}$. No thin polymer-layer coats on the front surface for acoustic impedance matching to avoid possible change in output energy by degradation of the coating after a long operating period. The receiver is a 0.5 -in. flat-surface transducer. The distance from the transmitter to the fabric and to the receiver is 1 and 1.25 in., respectively.

\section{Electronics}

To maximize the speed of data acquisition, increase resolution of data, and reduce cost, an eight-channel electronics system was designed to process signal analogically, such as filtering, holding, gating, and integration. Figure 2 is a schematic diagram of the electronics of the ultrasonic fabric-defect detection system. The transmitter is pulsed by a gated sine wave with six cycles and 80-V amplitude. After passing through three stages of preamps and a fourth-order Bessel band-pass filter, the receiving signals go into a fullwave rectifier. A switched integrator integrates the rectified signal of the first received pulse train inside an analog gate and holds it for data acquisition by the DSP device.

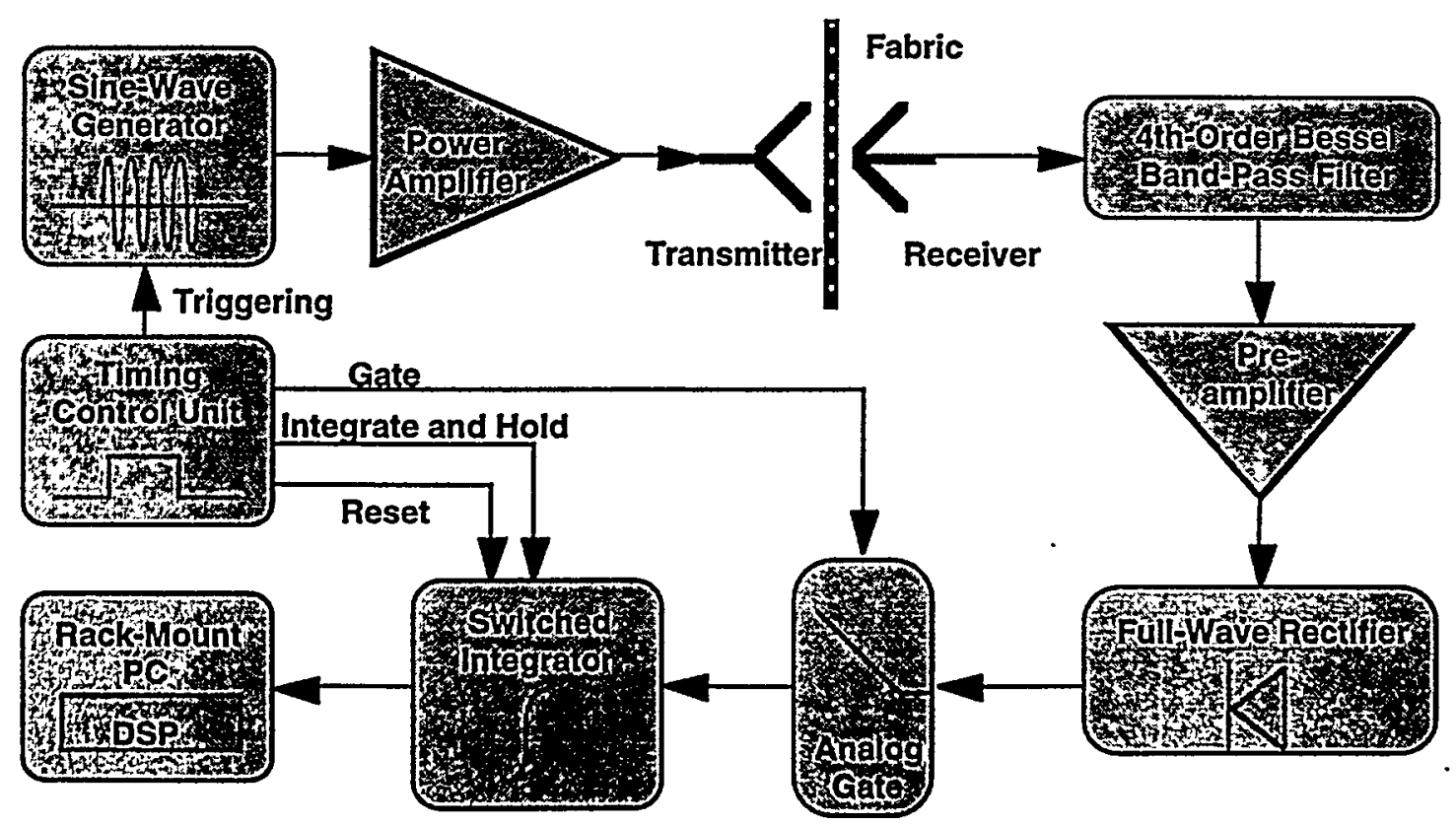

Figure 2. Schematic diagram of electronics of ultrasonic fabric-defect detection system. 
At a scanning speed of $10 \mathrm{in} . / \mathrm{sec}$ and a repetition rate of 2000 pulses/sec, the scanning resolution is 200 pixels/in. enough to cover every yarn in the warp direction. The data acquisition rate drops to 2000/channel which can be easily handled by any high resolution A/D converter or DSP board. The total required time and data size for image processing drops dramatically to approximately $1 / 500$ of those for full radio-frequency (RF) signals and the cost also drops to $1 / 15$ if high speed A/D boards are used.

\section{Scanning Mechanism}

The scanning mechanism uses transducer mounting devices, scanning rails, and the scanning device. Each transducer mounting device carries four transducers and is designed to provide flexible adjusting for focusing and alignment of transducer pairs. Each device is mounted on 8-feet long rod carried by two rollers that move on a low-cost, highly durable scanning rail. The front and back rods, which carry transmitters and receivers, respectively, are linked and are pushed back and forth by the scanning device. Because of the strong vibration and differing vibration modes generated by the weaving machine, a hollow-steel rectangular frame is mounted under each rail to provide extra strength.

To perform $100 \%$ inspection of the fabric under various feeding speeds and yarn sizes, the transducer array must scan back and forth along the pick direction at a speed of at .. least $10 \mathrm{in./sec.} \mathrm{Two} \mathrm{scanning} \mathrm{mechanisms,} \mathrm{a} \mathrm{computer} \mathrm{controlled} \mathrm{step} \mathrm{motor} \mathrm{and} \mathrm{an} \mathrm{AC-}$ motor-driven rotary system, were tested. The first has the advantages of being very easy to set up, well-controlled linear speed, and very accurate location measurements; however, it is expensive and difficult to operate independently without a computer. The second scanning mechanism is inexpensive, capable of running independently, and requires minimum maintenance. However, it is not easy to fine-tune the scanning speed and to accurately measure location because the $\mathrm{AC}$ motor does not have very stable output and the speed of the rotary disk is sinusoidal rather than linear. A better measurement of location can be achieved by putting photodiodes on the left and right frames of the rotary system to trigger the DSP board for data acquisition.

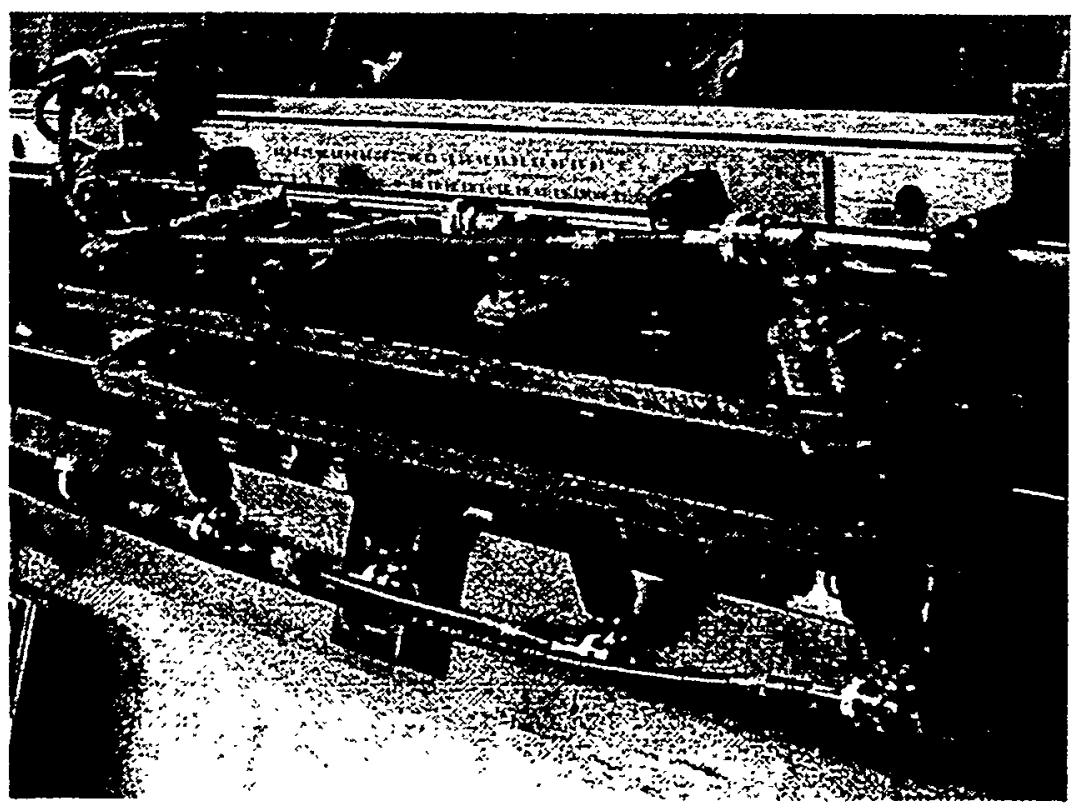

Figure 3. Details of transducer array mounting on scanning rails. 
Data Acquisition and Imaging Analysis

Because of the weaving speed and the resolution needed for on-loom defect detection, a large amount of data is generated. The PC31-20MIPS/50MFLOPS, a PC plug-in coprocessor with a 32-bit supercontroller, that runs independently once the software is loaded onto its on-board memory, synchronizes the AC motor, the electronics, and data acquisition. Equipped with a $20 \mathrm{MHz} \mathrm{A} / \mathrm{D}$ converter and a dual-port memory, capable of $50 \mathrm{MHz}$ hardware floating-point operation, the PC31 is powerful enough for real-time 2D image processing, defect analysis, and network communication to report defects. In addition, the DSP performs automatic baseline calibration and data averaging to improve the signal-to-noise ratio. Some simple image processing and defect detection algorithms, such as moving average, filtering, mean value, and standard deviation, are also implemented in the DSP software. Once a defect is detected and analyzed, information, such as size, location, orientation, and category, is put in a digital I/O buffer to wait for the central computer to download it through network without interrupting the DSP.

\section{DETECTION ALGORITHMS}

Because of limited memory for the executive program and acquired data, restricted processing time, and complexity of signals for certain defects, we cannot yet apply sophisticated algorithms such as neural networks, pattern recognition, and artificial intelligence their use may be possible when more powerful DSPs become available. The simple threshold checking of mean values and standard deviations along pick and warp directions can detect most pick and yarn defects. Localized defects can be detected by threshold checking after moving averaging and filtering. All thresholds are set or reset by the automatic baseline calibration whenever asked. The system is also continuously checks and updates the baseline and threshold values during weaving to correct any possible changes caused by electronics, temperature, and humidity. For easier detection, some warp and localized defects, such as endouts and reed marks, require extra high-pass filtering along pick, warp, or both directions to reduce background variation. During prototype evaluation, an image of 600 pixels $\times 30$ pixels, i.e. 5 -in. scanning along the pick direction during $30 \mathrm{sec}$ of weaving, is generated for each channel, and image processing and defect detection is performed accordingly.

\section{RESULTS OF ON-LOOM DEFECT DETECTION}

The defects are categorized into eight types: single pick, full-width multiple picks, warp, selvedge, oil/dirt, filling, variation in density or uniformity, and physically difficult to see. Each category has many kinds of defects, which are caused by various factors. The following sections demonstrate some results of detection and evaluation of several common, serious, or noncommon but serious defects from on-loom testing.

\section{Pick Defects}

Most of these defects are caused by problems of pick feeding or machine stops and starts. Figure 4 is a 600 pixels $\times 30$ pixels image of missing picks. The defect occurs when the pick yarn, shot from one side of the loom to the opposite side, is not caught by the catcher. These defects can easily be detected by checking the thresholds of the pick mean value and standard deviation. The pick mean value in Fig. 4 shows that there are three missing picks. Surprisingly, the pick mean value may either be reduced, increased, or both. Evaluation of the fabric and data shows that the receiving energy is increased when the ultrasonic beam hits a spot where a pick was missing and decreased when it hits the edge of the pick next to the spot. Figure 5 shows the image and detection of three double picks. This type of defect occur when the pick is double-folded during the air-jet process 

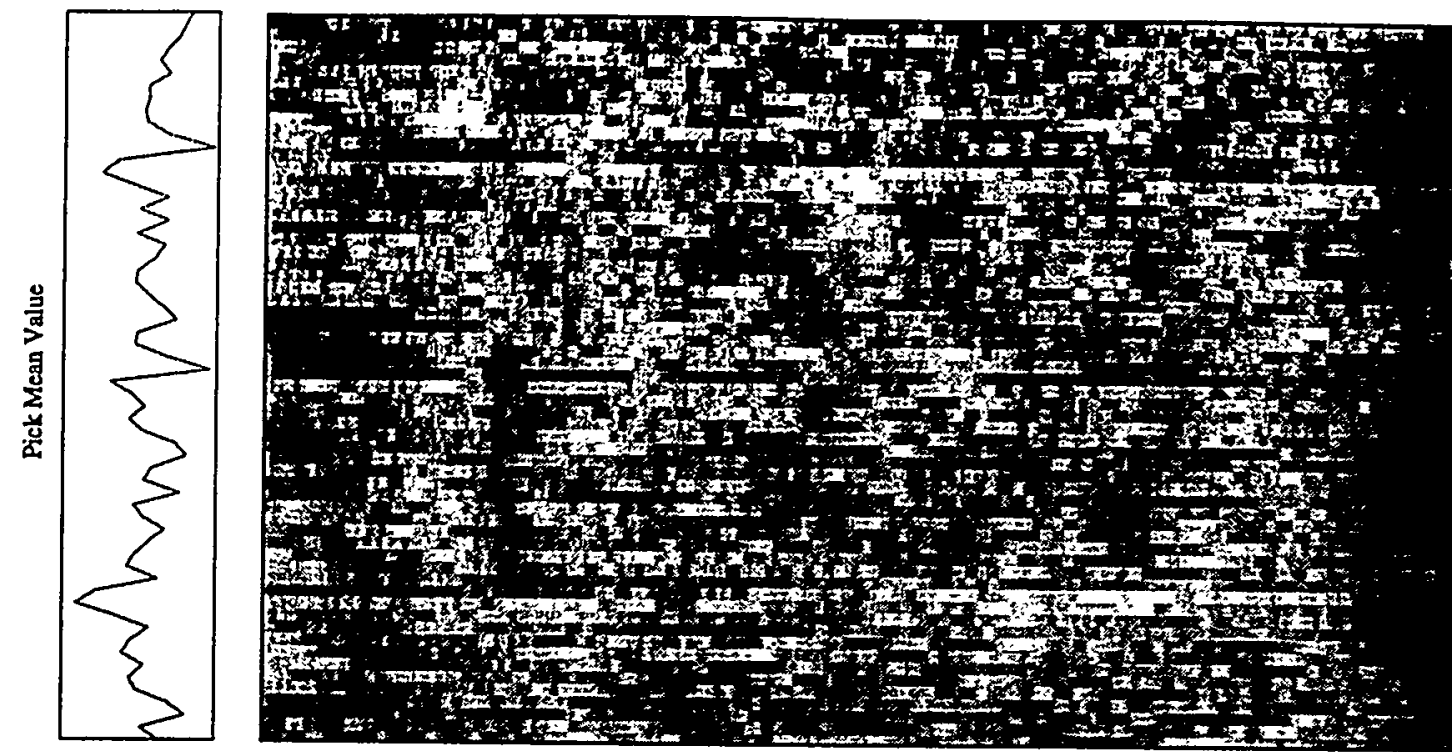

Figure 4. Ultrasonic image and pick mean value of three missing picks.
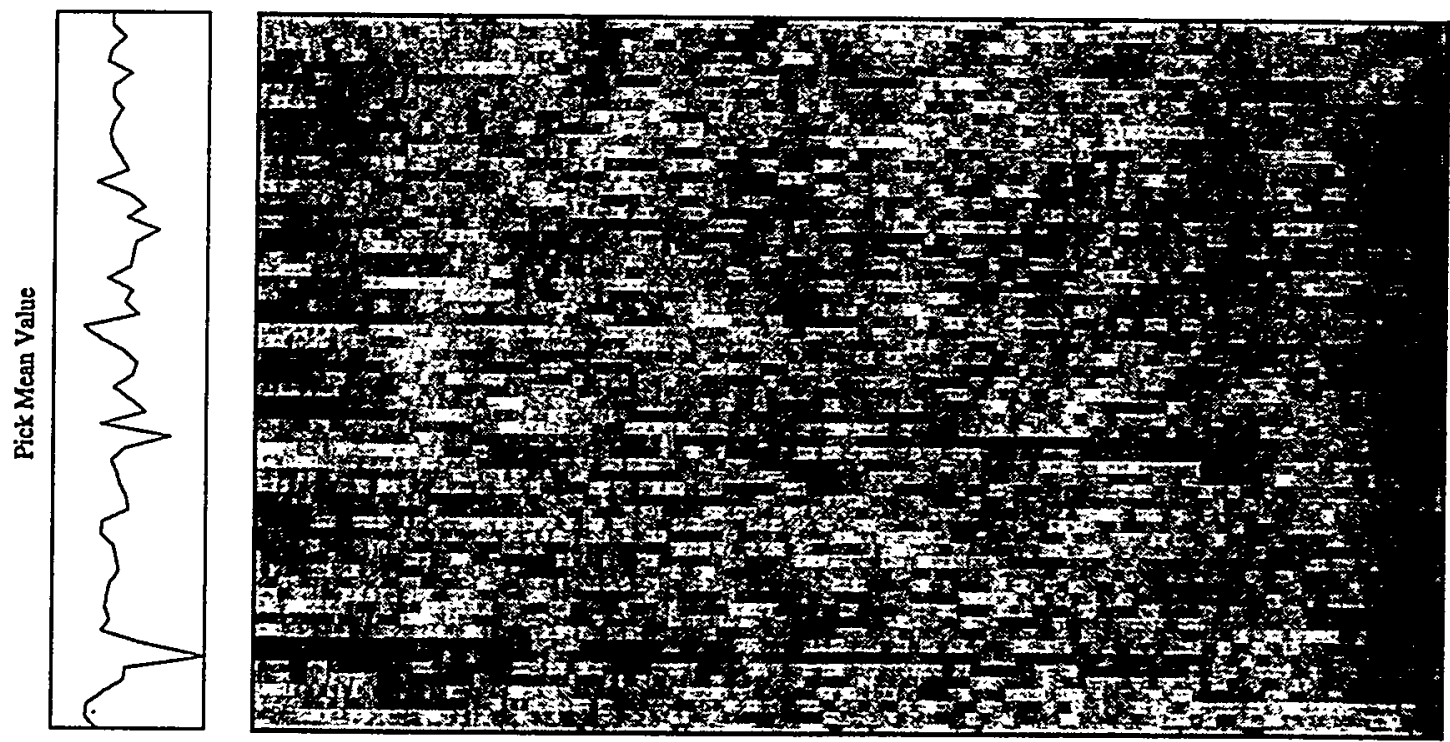

Figure 5. Ultrasonic image and pick mean value of three double picks.

and woven into the fabric. The pick mean value is always reduced because the defect doubles the thickness, i.e., increases the attenuation.

\section{Warp Defects}

Warp defects may be caused by changes in warp tension, broken yarn, or warp feeding problems. A 600 pixels $\times 30$ pixels ultrasonic image of a reed mark is shown in Fig. 6. This defect did not change the material but the warp tension changed during feeding. It is almost invisible during weaving unless one shines a flashlight on the fabric at 

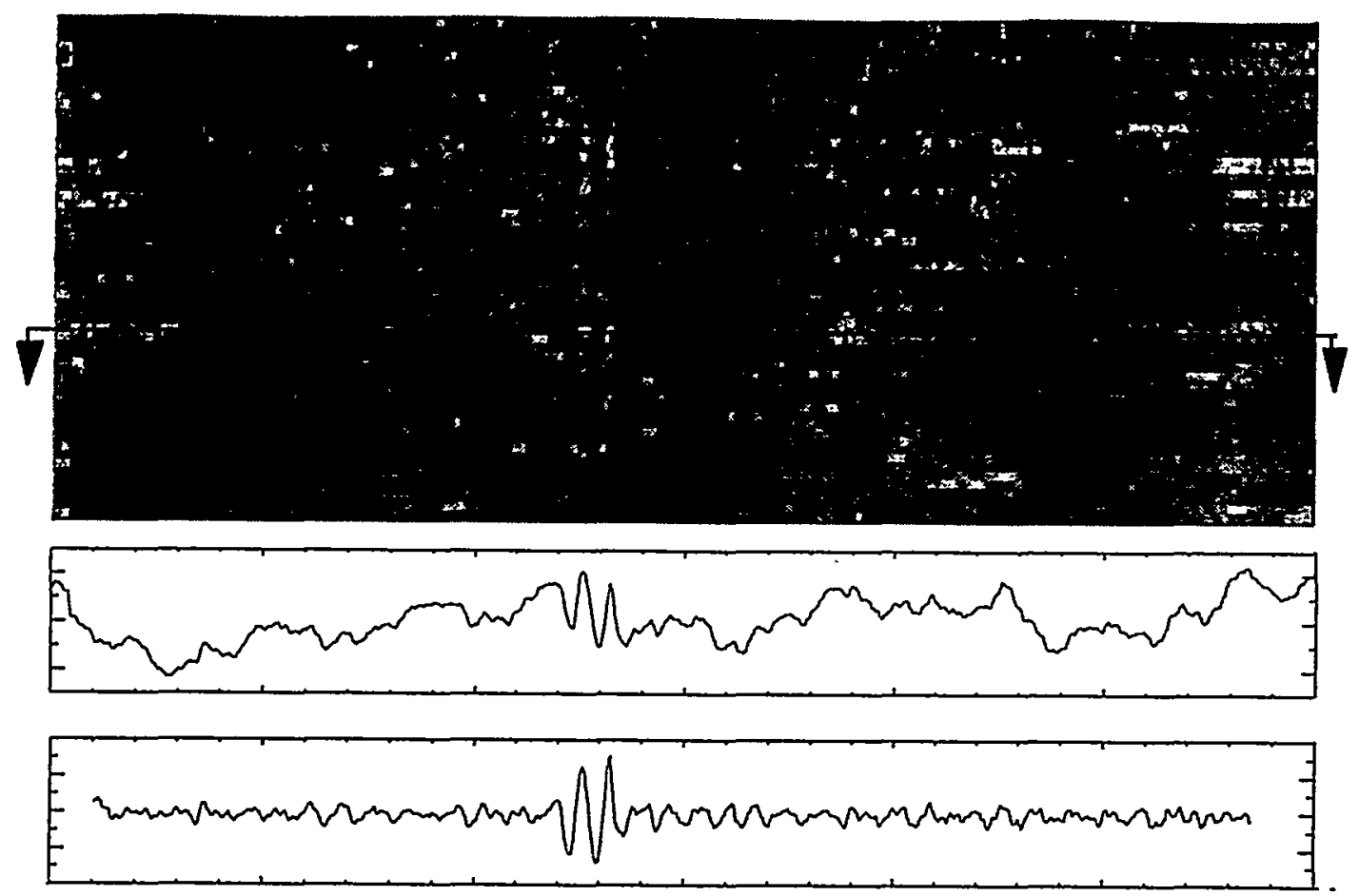

Figure 6. Ultrasonic image of reed mark and data in pick direction (along the dashed line) before and after high-pass filtering.

the correct angle. A warp defect will run continuously until the problem causes it is corrected. Therefore, early detection and alerting are very important so the operator can fix the problem promptly before more waste is produced. In the image, the defect is visible by eyes but cannot be detected by the mean value and standard deviation threshold methods. However, after simple and quick high-pass filtering along the pick direction, the defect stands out clearly and is easily detected. Figure 6 also shows the data along a pick before and after high-pass filtering. We are still developing algorithms for classifying and categorizing defects in real-time.

\section{Selvedge}

The selvedge protects the edge of the woven fabric so that it will not ravel. The signal intensity varies dramatically as scanning from normal woven, very dense selvedge area, thread, to air. Therefor, a pair of sensors, with different electronic gain setting, is operating independently from the transducer array to determine selvedge quality, either be normal, broken, too long, or too short. Figure 7 shows an image of a normal selvedge that contains threads of differing length.

\section{CONCLUSIONS}

A noncontact, on-loom ultrasonic inspection technique was developed for real-time $100 \%$ defect inspection of fabrics. A prototype was built and tested successfully on loom. The system is compact, rugged, low cost, requires minimal maintenance, is not sensitive to fabric color and vibration, and can easily be adapted to current loom configurations. Moreover, it can detect defects in both the pick and warp directions. The system is capable 


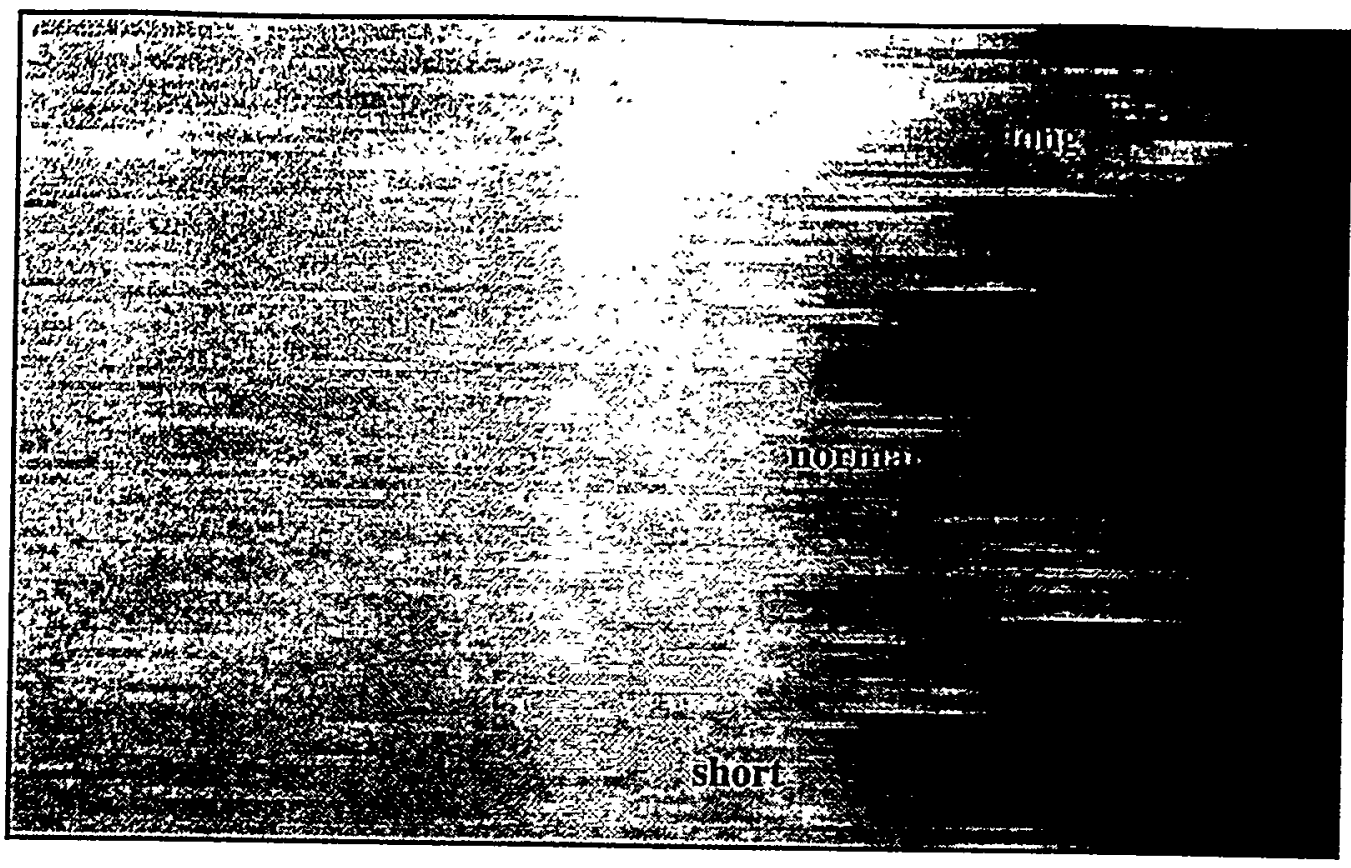

Figure 7. Ultrasonic image of long, short, and normal threads of selvedge.

of determining the size, location, and orientation of each defect. To further improve the system, air-coupled transducers with higher efficiency and sensitivity need to be developed. Advanced detection algorithms also need to be developed for better classification and categorization of defects in real-time.

\section{ACKNOWLEDGMENTS}

This work was supported by U.S. Department of Enèrgy, Office of Energy Research, Laboratory Technology Transfer Projects, under Contract W-31-109-ENG-38.

\section{REFERENCES}

1. S.-H. Sheen, H.-T. Chien, W. P. Lawrence, and A. C. Raptis, Ultrasonic Imaging System for In-Process Fabric Defect Detection, U.S. Patent No. 5,665,907, 1997. 